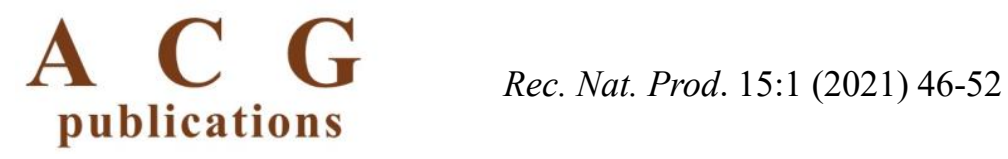

records of natural products

\title{
Two New Ecdysteroid Glycosides from the Rhizomes of
}

\section{Silene tatarinowii Regel}

\author{
Huawei Zhang $\oplus^{1,2^{*}}$, Yuzhu Tan $\oplus^{1}$ and Xiaoping Dong $\odot 1$ \\ ${ }^{1}$ School of Pharmacy, Chengdu University of Traditional Chinese Medicine, Chengdu 611137, China \\ ${ }^{2}$ School of Pharmacy, Shaanxi University of Chinese Medicine, Xianyang 712046, China
}

(Received July 10, 2020; Revised August 15, 2020; Accepted August 16, 2020)

\begin{abstract}
Two new ecdysteroid glycosides, sileneosides I and J (1 and 2), along with four known ecdysteroids (36) were isolated from the rhizomes of Silene tatarinowii Regel. Their structures were determined based on physicochemical properties and spectroscopic methods. In addition, the cytotoxicity of compounds 1-6 was evaluated in vitro in human SGC790, HCT116, A549, and BEL7404 tumor cell lines. The results showed that compounds 1 and 2 exhibited weak cytotoxicity against SGC790 ( $\left(\mathrm{IC}_{50} 82.58 \pm 0.53 \mu \mathrm{M}\right)$ and A549 cells $(96.62 \pm$ $0.58 \mu \mathrm{M})$, respectively.
\end{abstract}

Keywords: Silene tatarinowii; ecdysteroids; sileneoside I; sileneoside J; cytotoxic activity. (C) 2020 ACG Publications. All rights reserved.

\section{Introduction}

The genus Silene L. (Caryophyllaceae family) consists of approximately 700 species extensively distributing in the temperate regions of the Northern Hemisphere [1]. Silene tatarinowii Regel, an annual herbaceous plant of genus Silene, was commonly used as folk medicines for the treatment of dropsy and rheumatism [2]. Previous studies had discovered the characteristic components such as ecdysteroids and triterpenoid saponins from 115 species of Silene genus [3-5]. Ecdysteroids have been considered as one of the most important chemotaxonomic markers for this genus [5], which mainly possess significant anti-inflammatory [6], antitumor [7], antibacterial or antioxidant activities [5]. As part of an ongoing search for bioactive constituents from the traditional folk herbal medicines [8], two new ecdysteroid glycosides, sileneosides I and J (1 and 2), and four known ones: stachysterone A (3) [9], 22-O-acetyl-20-hydroxyecdysone (4) [10], sidisterone (5) [11], 22-oxo-20-hydroxy-ecdysone (6) [12] (Figure 1), were isolated from the rhizomes of S. tatarinowii. This study aims to describe the structure identification and the cytotoxic activities of these compounds.

\section{Materials and Methods}

\subsection{General Experimental Procedures}

ESI-MS was performed on Waters Quattro Premier instrument. The HR-ESI-MS spectra were taken on an Agilent Technologies 6550 Q-TOF. 1D and 2D NMR spectra were recorded on a BrukerAVANCE 400 instrument with TMS as an internal standard. Gas chromatography was taken on an Agilent Technologies 7890A. Semipreparative HPLC was performed on a system comprising an LC-

${ }^{*}$ Corresponding author: E-Mail: 307838553@qq.com (Huawei Zhang). 
6AD pump equipped with a SPD-20A UV detector and a Ultimate $\mathrm{XB}^{-\mathrm{C}_{18}}(10 \mathrm{~mm} \times 250 \mathrm{~mm}, 5 \mu \mathrm{m}$ particles). Silica gel was purchased from Qingdao Haiyang Chemical Group Corporation (Qingdao, China). MCI gel column were purchased from Mitsubishi Chemical Holdings. Sephadex LH-20 gel silica gel was purchased from GE Healthcare Bio-Sciences AB.

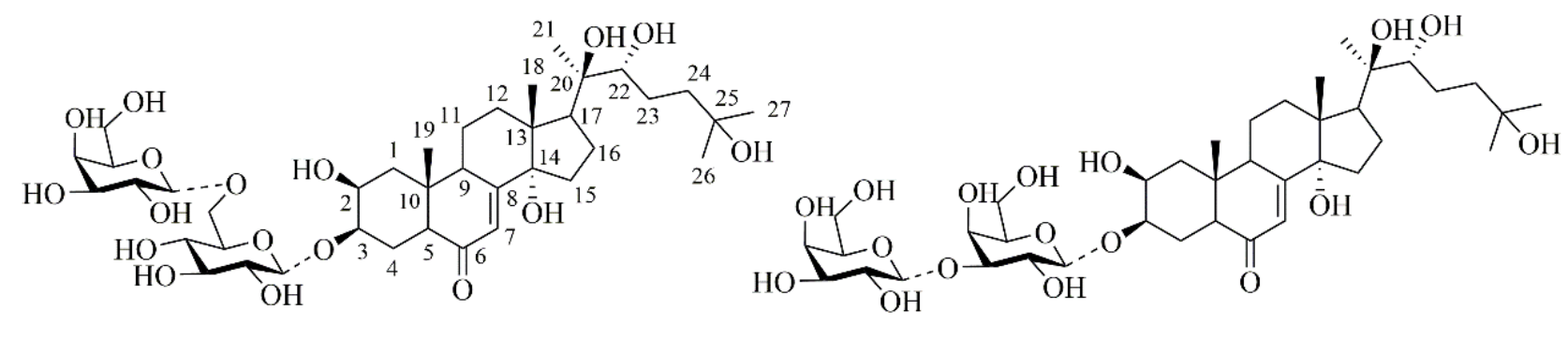<smiles>CC(C)(O)CC[C@@H](O)[C@@](C)(O)C1CCC2(C)C3=CC(=O)C4CC(O)C(O)CC4C3CC=C12</smiles>

3<smiles>C[C@@]12C[C@H](O)[C@@H](O)CC1C(=O)C=C1C2CC[C@]2(C)[C@@H]([C@H]3C=CC(=O)O3)CC[C@@H]12</smiles>

5<smiles>CC(=O)O[C@H](CCC(C)(C)O)[C@](C)(O)[C@H]1CC[C@H]2C3=CC(=O)C4C[C@@H](O)[C@@H](O)C[C@]4(C)C3CC[C@]12C</smiles>

4<smiles>CC(C)(O)CCC(=O)[C@H](O)C1CCC2C3=CC(=O)C4C[C@@H](O)[C@@H](O)CC4(C)C3CCC21C</smiles>

6

Figure 1. Structures of compounds 1

\subsection{Plant Material}

The dried rhizomes of S. tatarinowii Regel were collected in October 2018 from north slope of Qinling mountains in Shaanxi Province of China, and was identified by Associate Professor Yuzhu Tan (Chengdu University of Traditional Chinese Medicine). A voucher specimen (No. 20181010) was deposited at the Medicinal Plants Herbarium (MPH), Chengdu University of Traditional Chinese Medicine, Chengdu, China.

\subsection{Extraction and Isolation}

The powdered rhizomes of $S$. tatarinowii Regel $(3.8 \mathrm{~kg})$ were extracted three times by $70 \% \mathrm{EtOH}$ under the condition of $80{ }^{\circ} \mathrm{C}$ reflux to obtain a crude extract $(160.3 \mathrm{~g})$. The extraction was suspended in water, and then was sequentially extracted with $n$ - BuOH. The $n$-BuOH layer residue $(57.6 \mathrm{~g})$ was subjected to silica gel column chromatography $\left(\mathrm{CHCl}_{3}: \mathrm{MeOH}\right.$ mixture 15:1 to 3:1, v/v), to yield four fractions (Fr. 1-Fr. 4). Fr. 2 (18.9 g) was decolorized on a MCI gel column (MeOH: $\mathrm{H}_{2} \mathrm{O}$ mixture (8:1 to 1:1, v/v)), to yield three subfractions (Fr. 2-1-Fr. 2-3). Fr. 2-1 (3.3 g) was purified over Sephadex LH20 column $(\mathrm{MeOH})$, and then by semi-preparative HPLC eluted with $\mathrm{MeOH}: \mathrm{H}_{2} \mathrm{O}(21 \%$, v/v, $2 \mathrm{~mL} / \mathrm{min})$ to get compound $1\left(10 \mathrm{mg}, t_{\mathrm{R}}=33 \mathrm{~min}\right)$ and compound $\mathbf{2}\left(9 \mathrm{mg}, t_{\mathrm{R}}=36 \mathrm{~min}\right)$. Fr. 2-2 (3.9 g) was purified 
by semi-preparative HPLC eluted with $\mathrm{MeOH}: \mathrm{H}_{2} \mathrm{O}(42 \%$, v/v, $2 \mathrm{~mL} / \mathrm{min})$ to afford compound 3 (4 $\mathrm{mg}$, $\left.t_{\mathrm{R}}=78 \mathrm{~min}\right)$, compound $4\left(8 \mathrm{mg}, t_{\mathrm{R}}=62 \mathrm{~min}\right)$, compound $5\left(5 \mathrm{mg}, t_{\mathrm{R}}=48 \mathrm{~min}\right)$, and compound $6(6$ $\left.\mathrm{mg}, t_{\mathrm{R}}=30 \mathrm{~min}\right)$.

\subsection{Spectroscopic Data}

Sileneoside I (1): A white amorphous powder, $[\alpha]_{\mathrm{D}}^{25} 48.5\left(c 0.2, \mathrm{CH}_{3} \mathrm{OH}\right) ; \mathrm{UV}\left(\mathrm{CH}_{3} \mathrm{OH}\right) \lambda \max : 232 \mathrm{~nm}$, $275 \mathrm{~nm}$; IR (KBr) $v_{\max }: 3393,2963,2940,1653,1379$ and $1057 \mathrm{~cm}^{-1}$; ${ }^{1} \mathrm{H}-\mathrm{NMR}$ (400 MHz, pyridine- $d_{5}$ ) and ${ }^{13} \mathrm{C}$-NMR (100 MHz, pyridine- $d_{5}$ ) spectral data, see Table 1; HR-ESI-MS: $m / z 827.4049[\mathrm{M}+\mathrm{Na}]^{+}$ (calcd for $\mathrm{C}_{39} \mathrm{H}_{64} \mathrm{O}_{17} \mathrm{Na}, 827.4041$ ).

Sileneoside J (2): A white amorphous powder, $[\alpha]_{\mathrm{D}}^{25} 47.8\left(c 0.2, \mathrm{CH}_{3} \mathrm{OH}\right) ; \mathrm{UV}\left(\mathrm{CH}_{3} \mathrm{OH}\right) \lambda \max : 230 \mathrm{~nm}$, $270 \mathrm{~nm}$; IR (KBr) $v_{\max }: 3393,2963,2940,1653,1379$ and $1057 \mathrm{~cm}^{-1}$; ${ }^{1} \mathrm{H}-\mathrm{NMR}$ (400 MHz, pyridine- $d_{5}$ ) and ${ }^{13} \mathrm{C}-\mathrm{NMR}\left(100 \mathrm{MHz}\right.$, pyridine- $d_{5}$ ) spectral data, see Table 1; HR-ESI-MS: $m / z 827.4034[\mathrm{M}+\mathrm{Na}]^{+}$ (calcd for $\mathrm{C}_{39} \mathrm{H}_{64} \mathrm{O}_{17} \mathrm{Na}, 827.4041$ ).

\section{Results and Discussion}

\subsection{Structure Elucidation}

Compound 1 was a white amorphous powder, showing maximum UV absorption at $232 \mathrm{~nm}$. The IR spectrum of 1 showed typical absorption bands for $\mathrm{OH}\left(3393 \mathrm{~cm}^{-1}\right)$ and conjugated $\mathrm{C}=\mathrm{O}(1653$ $\mathrm{cm}^{-1}$ ), corresponding to common characteristics of ecdysteroids [13]. It was appointed the molecular formula was $\mathrm{C}_{39} \mathrm{H}_{64} \mathrm{O}_{17}$ via the HR-ESI-MS positive ion at $\mathrm{m} / z$ 827.4049 $\left[\mathrm{M}+\mathrm{Na}{ }^{+}\right.$(calcd for $\mathrm{C}_{39} \mathrm{H}_{64} \mathrm{O}_{17} \mathrm{Na}, 827.4041$ ). The ${ }^{1} \mathrm{H}-\mathrm{NMR}$ spectrum (Table 1) of 1 showed five methyl protons at $\delta_{\mathrm{H}} 1.22$ (3H, s, Me-18), 0.99 (3H, s, Me-19), 1.62 (3H, s, Me-21), 1.40 (3H, s, Me-26) and 1.40 (3H, s, Me-27), an olefinic proton $6.20(1 \mathrm{H}, \mathrm{s}, \mathrm{H}-7)$, as well as signals for two anomeric protons at $\delta_{\mathrm{H}} 5.56(\mathrm{~d}, J=3.0$ $\mathrm{Hz}$ ) and $\delta_{\mathrm{H}} 5.49(\mathrm{~d}, J=3.0 \mathrm{~Hz})$. The ${ }^{13} \mathrm{C}-\mathrm{NMR}$ spectrum (Table 1) displayed 39 carbon signals, $27 \mathrm{of}$ which belonged to the aglycone carbons, while the remaining signals were assignable to one glucopyranosyl moiety $\left(\delta_{\mathrm{C}} 104.4,73.0,80.5,72.1,79.8,68.7\right)$ and one galactopyranosyl moiety $\left(\delta_{\mathrm{C}}\right.$ $101.0,71.4,71.8,71.4,72.4,63.0)$. Among the carbon signals of the aglycone, $\delta_{\mathrm{C}} 18.4,22.0,24.8,30.5$ and 30.6 were due to five methyl groups, $\delta_{\mathrm{C}} 203.6,167.1$ and 122.0 were due to an $\alpha, \beta$-unsaturated ketone group [14]. In comparison of the proton and carbon data, the aglycone resonances of 1 were almost consistent with those of 20-hydroxyecdysone [15], the difference was that the chemical shift of C-3 was transfered to downfield at $\delta_{\mathrm{C}} 80.1\left(\triangle \delta_{\mathrm{C}}+11.6\right)$, indicating that the glycosidation positions was at $\mathrm{C}$-3. In detail, in the $\mathrm{HMBC}$ spectrum (Figure 2), the correlation between the proton signal at $\delta_{\mathrm{H}} 2.76$ (H-5) with the carbon signal at $\delta_{\mathrm{C}} 204.3$, suggested the location of the keto group at C-6, which was further confirmed by the singlet proton at $\delta_{\mathrm{H}} 6.20(\mathrm{H}-7)$. These data allowed us to recognize the aglycone of compound 1 as a cholest-7en-6-one derivative. HMBC (Figure 2) correlations of H-21/C-17 and C20, H-22/C-20, C-21, C-23 and C-24, H-26/C-24, C-25 and C-27, indicated that the appearance of an isooctane group linked at $\mathrm{C}-17$ position. Moreover, $\mathrm{HMBC}$ correlations of $\mathrm{H}-1 / \mathrm{C}-3$ and $\mathrm{C}-5, \mathrm{H}-7 / \mathrm{C}-5$, C-9 and C-14, H-17/C-13, C-18 and C-21, H-18/C-12, C-13, C-14 and C-17, H-19/C-1, C-5, C-9 and $\mathrm{C}-10$, were assigned. In addition, HMBC correlations of H-Gal-1/C-Glc-6 and H-Glc-1/C-3 disclosed that the terminal galactopyranosyl unit was linked at C-6 of the inner glucopyranosyl unit, then the glucopyranosyl unit was linked at C-3 of the aglycone. The glucopyranosyl moiety and galactopyranosyl moiety were identified as D-glucopyranose and D-galactopyranose by acid hydrolysis of $\mathbf{1}$, followed by TLC comparison with a reference compound and the optical rotation determination $\left([\alpha]_{\mathrm{D}}^{20}+49.2(c 0.16\right.$, $\left.\left.\mathrm{H}_{2} \mathrm{O}\right), \quad[\alpha]_{\mathrm{D}}^{20}+80.1\left(c 0.14, \mathrm{H}_{2} \mathrm{O}\right)\right)[16]$, and judged to be in $\alpha$-configuration from the coupling constants of two anomeric protons ( $3.0 \mathrm{~Hz}$ each). In the NOESY spectrum of 1 (Figure 2), the NOE correlations of $\mathrm{H}-4 / \mathrm{H}-2 / \mathrm{H}-9, \mathrm{H}-1 \mathrm{a} / \mathrm{H}-5$ and $\mathrm{Me}-19$, indicated $\beta$-orientations of $2-\mathrm{OH}$ and $5-\mathrm{H}$, which supported the $\mathrm{A} / \mathrm{B}$ cis ring junction pattern; the NOE correlations of $\mathrm{H}-12 \mathrm{a} / \mathrm{H}-15 \mathrm{a} / \mathrm{H}-16 \mathrm{a}$ and $\mathrm{Me}-18, \mathrm{H}-12 \mathrm{~b} / \mathrm{H}-17$, supported the $\mathrm{C} / \mathrm{D}$ trans ring junction pattern; and the NOE correlations of $\mathrm{H}-12 \mathrm{~b} / \mathrm{Me}-21$ and $\mathrm{H}-22 / \mathrm{H}-$ 16a, suggested $\alpha$-axial configurations of $\mathrm{Me}-21$ and $22-\mathrm{OH}$, and $\beta$-orientations of $20-\mathrm{OH}$. Therefore, compound 1 was identified as $2 \beta, 3 \beta, 14 \alpha, 20 R, 22 R, 25$-hexahydroxy- $5 \beta$-cholest-7-en-6-on-3-

$O$-[ $\alpha$-D-galactopyranosyl-( $1 \rightarrow 6)-\alpha$-D-glucopyranosyl], which was named as sileneoside I. 
Table 1. ${ }^{1} \mathrm{H}-\mathrm{NMR}\left(400 \mathrm{MHz}\right.$, in pyridine- $\left.d_{5}\right)$ and ${ }^{13} \mathrm{C}-\mathrm{NMR}\left(100 \mathrm{MHz}\right.$, in pyridine- $\left.d_{5}\right)$ spectral data of compounds 1 and 2

\begin{tabular}{|c|c|c|c|c|}
\hline \multirow{2}{*}{ No. } & \multicolumn{2}{|r|}{1} & \multicolumn{2}{|r|}{2} \\
\hline & $\delta \mathrm{c}$ & $\delta_{\mathrm{H}}(J$ in $\mathrm{Hz})$ & $\delta \mathrm{c}$ & $\delta_{\mathrm{H}}(J$ in $\mathrm{Hz})$ \\
\hline \multirow{2}{*}{1} & \multirow{2}{*}{39.9} & $2.12(\mathrm{~m}, \mathrm{H}-1 \mathrm{a})$ & \multirow{2}{*}{39.0} & $2.13(\mathrm{~m}, \mathrm{H}-1 \mathrm{a})$ \\
\hline & & $1.75(\mathrm{~m}, \mathrm{H}-1 \mathrm{~b})$ & & $1.73(\mathrm{~m}, \mathrm{H}-1 \mathrm{~b})$ \\
\hline 2 & 68.5 & $4.06, \mathrm{~m}$ & 68.6 & $4.06, \mathrm{~m}$ \\
\hline 3 & 80.1 & $4.02, \mathrm{~m}$ & 81.5 & $4.72, \mathrm{~m}$ \\
\hline \multirow{2}{*}{4} & \multirow{2}{*}{32.2} & $2.33(\mathrm{~m}, \mathrm{H}-4 \mathrm{a})$ & \multirow{2}{*}{32.2} & $2.13(\mathrm{~m}, \mathrm{H}-4 \mathrm{a})$ \\
\hline & & $1.85(\mathrm{~m}, \mathrm{H}-4 \mathrm{~b})$ & & $1.86(\mathrm{~m}, \mathrm{H}-4 \mathrm{~b})$ \\
\hline 5 & 53.0 & $2.76(1 \mathrm{H}, \mathrm{dd}, 3.0,10.1)$ & 52.9 & $2.67(1 \mathrm{H}, \mathrm{dd}, 2.4,12.6)$ \\
\hline 6 & 204.3 & - & 203.4 & - \\
\hline 7 & 122.1 & $6.20, \mathrm{~s}$ & 122.0 & $6.21, \mathrm{~s}$ \\
\hline 8 & 167.8 & - & 167.1 & - \\
\hline 9 & 34.8 & $3.50(1 \mathrm{H}, \mathrm{t}, 8.3)$ & 34.7 & $3.47(1 \mathrm{H}, \mathrm{t}, 8.2)$ \\
\hline 10 & 39.1 & - & 39.8 & - \\
\hline \multirow{2}{*}{11} & \multirow{2}{*}{21.5} & $1.82(\mathrm{~m}, \mathrm{H}-11 \mathrm{a})$ & \multirow{2}{*}{21.5} & $1.76(\mathrm{~m}, \mathrm{H}-11 \mathrm{a})$ \\
\hline & & 1.67 (m, H-11b) & & $1.66(\mathrm{~m}, \mathrm{H}-11 \mathrm{~b})$ \\
\hline \multirow{2}{*}{12} & \multirow{2}{*}{32.4} & $2.60(\mathrm{~m}, \mathrm{H}-12 \mathrm{a})$ & \multirow{2}{*}{32.4} & $2.55(\mathrm{~m}, \mathrm{H}-12 \mathrm{a})$ \\
\hline & & $2.01(\mathrm{~m}, \mathrm{H}-12 \mathrm{~b})$ & & 1.98 (m, H-12b) \\
\hline 13 & 48.6 & - & 48.5 & - \\
\hline 14 & 84.6 & - & 84.6 & - \\
\hline \multirow{2}{*}{15} & \multirow{2}{*}{32.2} & $2.10(\mathrm{~m}, \mathrm{H}-15 \mathrm{a})$ & \multirow{2}{*}{32.4} & $2.13(\mathrm{~m}, \mathrm{H}-15 \mathrm{a})$ \\
\hline & & $1.85(\mathrm{~m}, \mathrm{H}-15 \mathrm{~b})$ & & $1.76(\mathrm{~m}, \mathrm{H}-15 \mathrm{~b})$ \\
\hline \multirow{2}{*}{16} & \multirow{2}{*}{22.2} & 2.45 (m, H-16a) & \multirow{2}{*}{22.2} & 2.44 (m, H-16a) \\
\hline & & $2.10(\mathrm{~m}, \mathrm{H}-16 \mathrm{~b})$ & & 2.09 (m, H-16b) \\
\hline 17 & 50.6 & $3.02(1 \mathrm{H}, \mathrm{t}, 8.1)$ & 50.6 & $2.97(1 \mathrm{H}, \mathrm{t}, 8.4)$ \\
\hline 18 & 18.4 & $1.22(\mathrm{~s}, 3 \mathrm{H})$ & 18.4 & $1.20(\mathrm{~s}, 3 \mathrm{H})$ \\
\hline 19 & 24.8 & $0.99(\mathrm{~s}, 3 \mathrm{H})$ & 24.7 & $0.93(\mathrm{~s}, 3 \mathrm{H})$ \\
\hline 20 & 77.5 & - & 77.4 & - \\
\hline 21 & 22.0 & $1.62(\mathrm{~s}, 3 \mathrm{H})$ & 22.0 & $1.59(\mathrm{~s}, 3 \mathrm{H})$ \\
\hline 22 & 78.1 & $3.90, \mathrm{~m}$ & 78.1 & $3.86, \mathrm{~m}$ \\
\hline \multirow{2}{*}{23} & \multirow{2}{*}{28.0} & $2.18(\mathrm{~m}, \mathrm{H}-23 \mathrm{a})$ & \multirow{2}{*}{28.0} & $2.13(\mathrm{~m}, \mathrm{H}-23 \mathrm{a})$ \\
\hline & & 1.85 (m, H-23b) & & 1.81 (m, H-23b) \\
\hline \multirow{2}{*}{24} & \multirow{2}{*}{43.2} & $2.33(\mathrm{~m}, \mathrm{H}-24 \mathrm{a})$ & \multirow{2}{*}{43.2} & $2.29(\mathrm{~m}, \mathrm{H}-24 \mathrm{a})$ \\
\hline & & $1.85(\mathrm{~m}, \mathrm{H}-24 \mathrm{~b})$ & & $1.81(\mathrm{~m}, \mathrm{H}-24 \mathrm{~b})$ \\
\hline 25 & 70.2 & & 70.1 & - \\
\hline 26 & 30.7 & $1.40(\mathrm{~s}, 3 \mathrm{H})$ & 30.6 & $1.36(\mathrm{~s}, 3 \mathrm{H})$ \\
\hline 27 & 30.5 & $1.40(\mathrm{~s}, 3 \mathrm{H})$ & 30.5 & $1.36(\mathrm{~s}, 3 \mathrm{H})$ \\
\hline
\end{tabular}


Table 1 continued..

\begin{tabular}{|c|c|c|c|c|}
\hline Glc-1 & 104.4 & $5.49(1 \mathrm{H}, \mathrm{d}, 3.0)$ & & \\
\hline 2 & 73.0 & $4.73, \mathrm{~m}$ & & \\
\hline 3 & 80.5 & $4.63, \mathrm{~m}$ & & \\
\hline 4 & 72.1 & $4.51, \mathrm{~m}$ & & \\
\hline 5 & 79.8 & $4.50, \mathrm{~m}$ & & \\
\hline 6 & 68.7 & $\begin{array}{l}4.59(\mathrm{~m}, \mathrm{H}-6 \mathrm{a}) \\
4.25(\mathrm{~m}, \mathrm{H}-6 \mathrm{~b})\end{array}$ & & \\
\hline Gal-1' & 101.0 & $5.56(1 \mathrm{H}, \mathrm{d}, 3.0)$ & 104.2 & $5.77(1 \mathrm{H}, \mathrm{d}, 3.6)$ \\
\hline $2^{\prime}$ & 71.4 & $4.67, \mathrm{~m}$ & 71.3 & $4.70, \mathrm{~m}$ \\
\hline $3^{\prime}$ & 71.8 & $4.79, \mathrm{~m}$ & 79.9 & $4.06, \mathrm{~m}$ \\
\hline $4^{\prime}$ & 71.4 & 4.67, m & 71.8 & $4.50, \mathrm{~m}$ \\
\hline $5^{\prime}$ & 72.4 & $4.63, \mathrm{~m}$ & 73.1 & $4.70, \mathrm{~m}$ \\
\hline $6^{\prime}$ & 63.0 & $\begin{array}{l}4.42 \text { (m, H-6a) } \\
4.42 \text { (m, H-6b) }\end{array}$ & 61.3 & $\begin{array}{l}4.56(\mathrm{~m}, \mathrm{H}-6 \mathrm{a}) \\
4.28(\mathrm{~m}, \mathrm{H}-6 \mathrm{~b})\end{array}$ \\
\hline Gal-1" & & & 103.7 & $5.53(1 \mathrm{H}, \mathrm{d}, 1.6)$ \\
\hline $2 "$ & & & 71.8 & $4.54, \mathrm{~m}$ \\
\hline $3 "$ & & & 71.6 & $4.70, \mathrm{~m}$ \\
\hline $4 "$ & & & 71.8 & $4.54, \mathrm{~m}$ \\
\hline $5^{\prime \prime}$ & & & 73.9 & $4.90, \mathrm{~m}$ \\
\hline 6" & & & 63.1 & $\begin{array}{l}4.44 \text { (m, H-6a) } \\
4.31 \text { (m, H-6b) }\end{array}$ \\
\hline
\end{tabular}

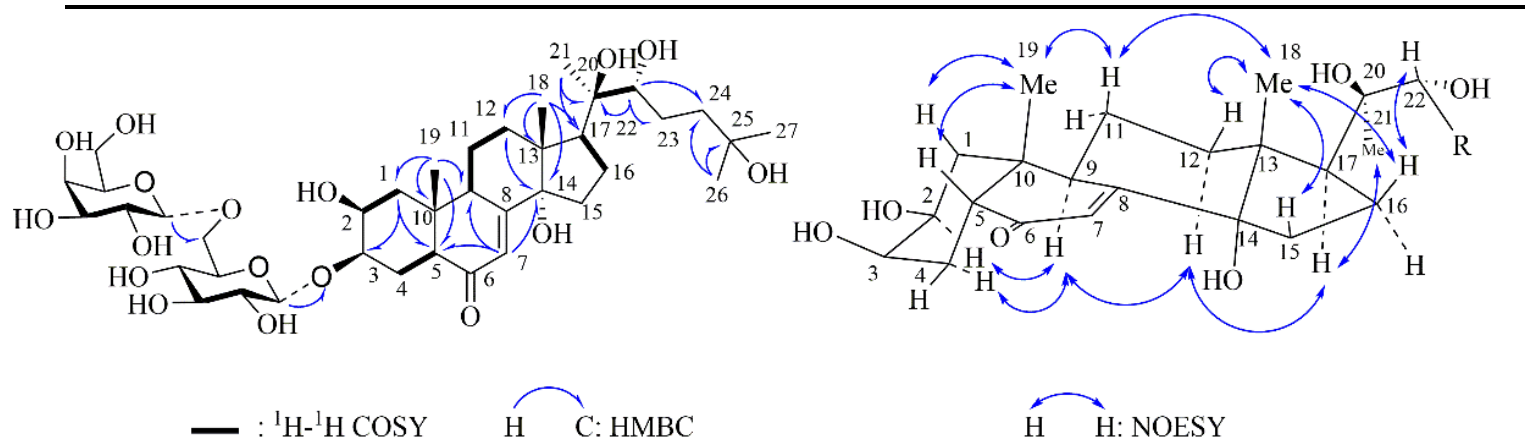

Figure 2. Key ${ }^{1} \mathrm{H}-{ }^{1} \mathrm{H}$ COSY, HMBC and NOESY correlations of compound 1

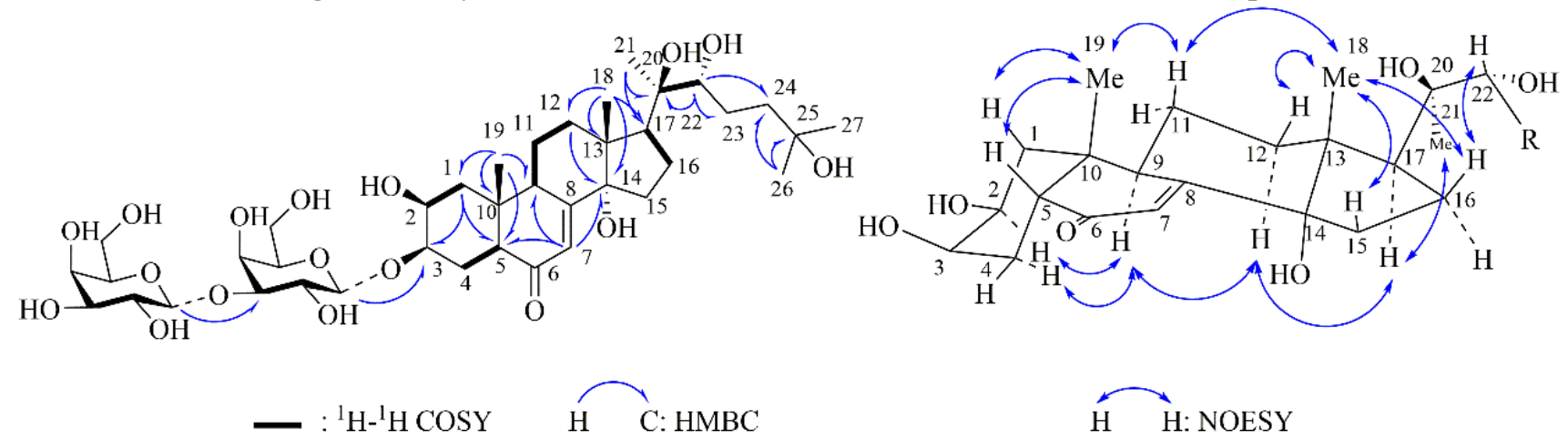

Figure 3. Key ${ }^{1} \mathrm{H}-{ }^{1} \mathrm{H}$ COSY, HMBC and NOESY correlations of compound 2 


\subsection{Cytotoxicity Assay}

The cytotoxic activity assay toward the SGC790, HCT116, A549, and BEL7404 tumor cell lines were measured by the MTT method in vitro, using 5-fluorouracil as positive control. Briefly, $1 \times 10^{4}$ $\mathrm{mL}^{-1}$ cells were seeded into 96-well plates and allowed to adhere for $24 \mathrm{~h}$. Compounds 1-6 were dissolved in DMSO and diluted with complete medium to six degrees of concentration (from 0.001 $\mathrm{mmol} / \mathrm{L}^{-1}$ to $0.4 \mathrm{mmol} / \mathrm{L}^{-1}$ ) for inhibition rate determination. After incubation at $37{ }^{\circ} \mathrm{C}$ for $4 \mathrm{~h}$, the supernatant fraction was removed before adding DMSO $(100 \mu \mathrm{L})$ to each well. The inhibition rate (IR) and $\mathrm{IC}_{50}$ were calculated (see Table 2). Values are mean $\pm \mathrm{SD}, \mathrm{n}=3$.

Table 2. Cytotoxicity of compounds 1-6 against four human cancer cell lines in vitro $\left(\mathrm{IC}_{50}, \mu \mathrm{M}\right)^{\mathrm{a}}$

\begin{tabular}{ccccc}
\hline Compounds & \multicolumn{4}{c}{ Cell lines } \\
\cline { 2 - 5 } & SGC790 & HCT116 & A549 & BEL7404 \\
\hline${ }^{\mathrm{b}} 5-\mathrm{Fu}$ & $8.4 \pm 1.9$ & $14.3 \pm 2.1$ & $8.0 \pm 1.6$ & $9.0 \pm 2.5$ \\
$\mathbf{1}$ & $82.58 \pm 0.53$ & $>100$ & $>100$ & $>100$ \\
$\mathbf{2}$ & $>100$ & $>100$ & $96.62 \pm 0.58$ & $>100$ \\
$\mathbf{3}$ & $>100$ & $>100$ & $>100$ & $>100$ \\
$\mathbf{4}$ & $>100$ & $>100$ & $>100$ & $>100$ \\
$\mathbf{5}$ & $>100$ & $>100$ & $>100$ & $>100$ \\
$\mathbf{6}$ & $>100$ & $>100$ & $>100$ & $>100$ \\
\hline
\end{tabular}

${ }^{\mathrm{a}} \mathrm{CC}_{50}$ values are means from three independent experiments (average $\pm \mathrm{SD}$ ) in which each compound concentration was tested in three replicate wells; ${ }^{\mathrm{b}} 5$-fluorouracil $(5-\mathrm{Fu})$ as positive control.

\section{Acknowledgments}

This project was financially supported by Shaanxi Provincial Science and Technology Department Project (grant number 2017JM8087)

\section{Supporting Information}

Supporting information accompanies this paper on http://www.acgpubs.org/journal/records-ofnatural-products

\section{ORCID}

Huawei Zhang: 0000-0003-4970-3818

Yuzhu Tan: 0000-0001-9698-2309

Xiaoping Dong: 0000-0001-7192-2131

\section{References:}

[1] N. Z. Mamadalieva, R. Lafont and M. Wink (2014). Diversity of secondary metabolites in the genus Silene L. (Caryophyllaceae) - structures, distribution, and biological properties, Diversity 6, 415-499.

[2] X. P. Hu (2016). Shaanxi provincial standards for medicinal materials. Shaanxi Science and Technology Publishing, Shaanxi, China,

[3] S. András, P. Zita, T. Gábor, M. Marianna, M. Imre and B. Mária (2004). A new ecdysteroid with unique $9 \beta-\mathrm{OH}$ and four other ecdysteroids from Silene italica ssp. Nemoralis, Steroids 69, 389-394.

[4] N. Takahashi, W. Li, and K. Koike (2016). Oleanane-type triterpenoid saponins from Silene armeria, Phytochemistry 129, 77-85.

[5] N. Z. Mamadalieva, L.N. Zibareva, R. Lafont, L. Dainan and Z. Saatov (2004). Phytoecdysteroids from the Silene genus, Chem. Nat. Compds. 40, 574-578.

[6] C. O. Ochieng, I. O. Ishola, S. A. Opiyo, L. A. Manguro, P. O. Owuor and K. C. Wong (2013). Phytoecdysteroids from the stem bark of Vitex doniana and their anti-inflammatory effects, Planta. Med. 79, 52-59.

[7] L. S. Yang, Y. H. Shen and F. Wang (2018). Study on the extraction and antitumor activity of Achyranthes Bidentata polysaccharide, Int. J. Biol. 10, 23-30. 
[8] H. W. Zhang, L. H. Zhou, C. Deng and G. L. Feng (2017). Antioxidant activity of the different polar parts from Dictamnus dasycarpus and its effects on tyrosinase activity, J. China. Pharm. 28, 4401-4404.

[9] W. T. Zhao, D. L. Meng, X. Li and W. Li (2007). Study on the chemical composition of Achyranthes Bidentata, J. Shenyang. Pharm. Univ. 4, 207-210.

[10] V. N. Odinokov, I. V. Galyautdinov, N. V. Nedopekin, L. M. Khalilov, A. S. Shashkov, V. V. Kachala, L. Dinan and R. Lafont (2002). Phytoecdysteroids from the juice of Serratula coronata L.(Asteraceae), Insect. Biochem. Molecul. 32, 161-165.

[11] J. P. Girault, M. Báthori, H. Kalász, I. Mathé and R. Lafont (1996). Sidisterone, a C 24 ecdysteroid from Silene dioica and Silene otitis,_J. Nat. Prod. 59, 522-524.

[12] C. Y. Tan, J. H. Wang, X. Li, Y. G. Du and X. F. Bai (2003). Chemical constituents of Cyanotis arachnoidea, Acta. Pharm. Sinica 38, 760-762.

[13] H. Attila, T. Gábor, S. András, M. Marianna, K. Zoltán, M. Imre and B. Mária (2004). Two new ecdysteroids from Serratula wolffii, J. Nat. Prod. 6, 1070-1072.

[14] Y. Meng, P. Whiting, S. Vladimir, H. H Rees and L. Dinan (2001). Limnantheoside C (20-Hydroxyecdysone 3 -O- $\beta$-D-glucopyranosyl-[1 $\rightarrow 3]-\beta$-D-xylopyranoside), a Phytoecdysteroid from Seeds of Limnanthes alba (Limnanthaceae), Z. Naturforsch. C, 56, 988-994.

[15] S. U. Rahman, A. Adhikari, M. Ismail, M. R. Shah and F. Ali (2017). A new trihydroxylated fatty acid and phytoecdysteroids from rhizomes of Trillium govanianum, Rec. Nat. Prod. 11, 323-327.

[16] C. S. Hudson, J. K. Dale (1917). Studies on the forms of d-glucose and their mutarotation, J. Am. Chem. Soc. 39, 320-328.

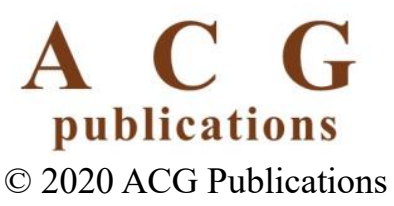

